\title{
Electrospinning of Guar Gum/Corn Starch Blends
}

\author{
Weiqiao Yanga,b, Ana MM Sousa ${ }^{a}$, Xihong Li ${ }^{\mathrm{b}}$, Peggy M Tomasula ${ }^{\mathrm{a}}$, LinShu Liu ${ }^{\mathrm{a} *}$ \\ ${ }^{a}$ Dairy and Functional Foods Research Unit, Eastern Regional Research Center, US Department of Agriculture, Wyndmoor, USA. \\ ${ }^{b}$ Key Laboratory of Food Nutrition and Safety (Ministry of Education), Tianjin University of Science and Technology, Tianjin, China.
}

Received: December 28, 2016; Accepted: January 05, 2017; Published: January 22, 2017

*Corresponding author: Lin Shu Liu, Dairy and Functional Foods Research Unit, Eastern Regional Research Center, ARS, US Department of Agriculture, 600 East Mermaid Lane, Wyndmoor, PA 19038, USA, Tel: +1(215)233-6486; E-mail: linshu.liu@ars.usda.gov

\begin{abstract}
In this study, electrospun nanofibers were prepared for the first time, from aqueous blends of guar gum (GG) and corn starch with amylose contents of $27.8 \%$ (CS28) and $50 \%$ (CS50). The fiber morphology and fiber diameter sizes (FDS) were correlated with solution rheology. The spinning solutions were prepared with $3 \mathrm{wt} \%$ total concentration and mass ratios ranging from $4: 1$ to $1: 4 \mathrm{GG} / \mathrm{CS}$. The $\mathrm{GG}$ alone (3 wt\%) was highly viscous and predominantly elastic $\left(G^{\prime}>G^{\prime \prime}\right)$ over the range of tested frequencies. Both CS were effective rheological modifiers that facilitated the electrospinning process. Partial substitution of GG by CS decreased solution viscosity and moved the elastic plateau $\left(G^{\prime}=G^{\prime \prime}\right)$ to higher frequencies resulting in improved fiber morphology and defectfree nanofibers with uniform FDS at an optimal GG/CS ratio of 2:1 for CS28 and of 1:1 for CS50. The sonication of CS50 prior to blending with GG was important to eliminate nanofiber defects. GG and CS are costattractive options to produce $100 \%$ food-grade electrospun nanofibers with potential to encapsulate active food ingredients and be used to develop functional foods and other active food systems.
\end{abstract}

Keywords: Nanofibers; Polysaccharide Blends; Amylose/ Amylopectin Ratio; Melojel; Hylon V; Rheology; Fiber Diameter Size;

\section{Introduction}

Electrospinning is a technology that produces continuous polymer nanofibers by application of high voltages to a polymer solution or melt [6]. Although the technology has been mainly applied to synthetic polymers its use in food applications has been increasing significantly $[1,28]$. This happens because electrospun nanofibers have small diameter sizes and very high surface area-to-mass ratios, typically exhibiting great functionality and improved mechanical performances when compared to fibers produced by other fiber-forming methods [6, 14]. Also, during electrospinning the solvent evaporates rapidly as the polymer jet travels towards the fiber collector which allows the production of fibers composed of polymer mixtures that would most likely phase separate if processed with other technologies [14]. Electrospun nanofibers and fibrous mats made from food-grade polymers or food-grade polymer mixtures can be used to develop functional foods, active food packaging and coatings by embedding active compounds in the fibers, to design foods with novel textures that provide enhanced satiety or even be used as processing aids and sensors $[5,14,19,28]$. The success of electrospinning will rely on the proper optimization of several process parameters including flow rate of spinning solution, applied voltage and distance from the needle tip to the fiber collector, as well as relevant solution properties such as viscosity, viscoelasticity, concentration, electrical conductivity and surface tension $[6,14,28]$.

Food-grade hydrocolloids of commercial importance include polysaccharides (natural carbohydrate polymers) and proteins. Food-grade hydrocolloids, alone or combined, are widely used by the food industry to improve texture, processing conditions and the overall quality of food products $[3,17]$. Processing hydrocolloids by electrospinning is often challenging since most of these compounds show inadequate or limited spinnability [28].

Starch and guar gum are two cost-attractive hydrocolloids often used together in food formulations [8]. Starch is present in plants as semi crystalline granules which shape, size, morphology and composition (e.g. amylose/amylopectin ratio) will depend on the starch source and cultivation conditions $[3,13]$. The granules are composed of amylose or $(1 \rightarrow 4)$-linked $\alpha$-glucopyranose, a linear macromolecule responsible for starch's gelling properties [18], and amylopectin or $(1 \rightarrow 4)$-linked $\alpha$-glucopyranose with $\alpha-(1 \rightarrow 6)$ branch linkages, a highly branched component that may form very weak gels [20]. Guar Gum (GG) is extracted from Cyampsis tetragonolobus seeds [32] and is formed by linear chains of $\beta$-(1-4)-D-mannan with side units of $\alpha-(1-6)$ linked galactose [24].

Electrospinning of starch and GG have been studied separately in a few papers. Lubambo et al. [16] found that fibers obtained from GG solutions were not uniform and had insoluble aggregates between fibers and within their structure. These authors concluded that sequential filtration steps were needed after GG purification to reduce the aggregates and improve fiber morphology. Pure starch fibers in turn, could only be obtained from Dimethyl Sulfoxide (DMSO) solutions using electro-wetspinning technology due to the non-volatile nature of the organic solvent $[12,13]$. Starch derivatives have also been electrospun from formic acid solutions $[15,34]$ while composite fibers have been produced from blends of starch with non-food grade polymers such as polyvinyl alcohol [29] and polylactic acid [30]. 
In this study, GG was mixed with two Corn Starches (CS) with different amylose contents $(27.8 \%$ and $50 \%)$ to produce, for the first time, electrospun GG/CS nanofibers using an environmentally friendly process. Uniform nanofibers were obtained from aqueous GG/CS blends without the need for either additional steps after GG purification or the use of any special additives or chemicals. The rheological properties of the GG, CS, and respective GG/CS blends were determined and related with fiber morphology and Fiber Diameter Size (FDS).

\section{Materials and methods}

\section{Materials}

Commercial guar gum (GG; lot \# SLBH5693V, India) was purchased from Sigma-Aldrich (St. Louis, MO, USA). Melojel corn starch with a reported $27.8 \%$ amylose content (CS28) and Hylon $\mathrm{V}$ corn starch with a reported $50 \%$ amylose content (CS50) were kindly supplied by Ingredion Incorporated (Bridgewater, N)). The respective average particle sizes (11.2 and $12.1 \mu \mathrm{m}$ ) and moisture contents ( 6.0 and $7.2 \%$ ) were determined in our laboratory. The mean particle sizes of CS28 and CS50 were determined by light scattering using a particle size meter (HORIBA Partica LA-950V2) while the moisture content was determined by drying a known amount of each CS in an air-oven at $120^{\circ} \mathrm{C}$ to constant weight. MilliQ water with a resistivity of $18.2 \mathrm{M} \Omega$. cm was prepared using Barnstead E-pure water system (Dubuque, IA) and used in all experiments.

\section{Methods}

\section{Preparation of spinning solutions}

Guar Gum (GG) was slowly dispersed in a vigorous vortex of Millipore water and left under moderate stirring overnight, at room temperature, to prepare a $\sim 1.3 \%$ wt solution. The solution was centrifuged in a Thermo Scientific centrifuge (Model Sorvall Legend X1R, Thermo Fisher Scientific Inc., Waltham, MA, USA) at $12,000 \mathrm{~g}$ and $40{ }^{\circ} \mathrm{C}$ for $1 \mathrm{~h}$. The supernatant was precipitated in ethanol at a 1:2 volume ratio followed by drying overnight in a vacuum oven to obtain the purified GG. A 3\% purified GG solution was then prepared by dissolving the galactomannan in Millipore water at $50 \stackrel{\circ}{\circ}$ for $3 \mathrm{~h}$ using an oil bath heated in a magnetic stirrer (model RCT basic, IKA, Werke Staufen, Germany) equipped with a PT 1000 IKA temperature sensor. .

Corn starch (CS) samples were dispersed in water under stirring at $85{ }^{\circ} \mathrm{C}$ for $1 \mathrm{~h}$ (CS28) and $95{ }^{\circ} \mathrm{C}$ for $1.5 \mathrm{~h}$ (CS50) to prepare CS dispersions with $3 \mathrm{wt} \%$ concentration. After heating, CS50 dispersions were sonicated for 10 min using an ultrasonic probe and 100\% amplitude (Cole Palmer Sonicator, model 130 $\mathrm{W}, 20 \mathrm{kV}$ ) equipped with a $1 / 2$ " microtip (constant duty cycle). The sonication was performed in the pulsed mode with the sonicator on for 30 s, then off for 30 s and the sample vessels were kept in an ice-water bath during treatment. Sonication time does not include the resting time.

GG and CS starting solutions were mixed at different mass ratios $(4: 1,2: 1,1: 1,1: 2$ and $1: 4)$ to prepare GG/CS blends with $3 \%$ wt total polysaccharide concentration. Mixing was carried out at $70{ }^{\circ} \mathrm{C}$ for $2 \mathrm{~h}$ under gentle stirring. Spinning solutions only composed of GG or CS (GG/CS mass ratios of 1:0 and 0:1, respectively) were submitted to the same thermal treatment as the blends. All samples were prepared in closed cap vials to prevent solvent evaporation.

\section{Rheological measurements}

The rheological tests were performed at $50^{\circ} \mathrm{C}$ using a Kinexus Rheometer (Malvern Instruments, Worcestershire, UK) with attached cone-plate geometry $\left(40 \mathrm{~cm}, 2^{\circ}\right.$ cone, truncation gap 54 $\mu \mathrm{m})$.

The GG/CS blends were loaded on the Peltier plate (preheated to $50{ }^{\circ} \mathrm{C}$ ) and immediately covered with paraffin oil to prevent water evaporation. Oscillatory measurements were carried out in the linear viscoelastic region of each sample, determined by strain sweep tests. After an equilibration step of 5 $\min$ at $50{ }^{\circ} \mathrm{C}$, the viscoelastic properties (elastic modulus, $\mathrm{G}^{\prime}$, and viscous modulus, $\left.G^{\prime \prime}\right)$ as function of frequency were measured through frequency sweep tests over the range, $0.01-10 \mathrm{~Hz}$. Steady shear measurements were performed in the shear rate range of 0.001-1000 $\mathrm{s}^{-1}$ to build flow curves from apparent viscosity data of each sample.

\section{Electrospinning}

GG/CS blends were prepared by electrospinning using a NaBond NEU-PRO unit (NaBond Technologies Co., Limited, China). A tubeless spinneret (NaBond Technologies Co., Limited, China) connected to an external syringe pump (Veryark, model TCI-IV) was attached inside a cabinet housing a drum collector. Each sample was loaded onto a $5 \mathrm{~mL}$ plastic syringe (initial sample volume of $3 \mathrm{~mL}$ ) with an attached metallic needle (outer diameter $\times$ length $=0.7 \mathrm{~mm} \times 32 \mathrm{~mm}$ and inner diameter $=0.390$ $\mathrm{mm}$ ) and placed in the spinneret. A scheme of the electrospinning set-up can be found elsewhere [26]. After several preliminary trials the electrospinning conditions were fixed at: $1 \mathrm{~mL} / \mathrm{h}$ of flow rate (Note: value set in the syringe pump matching a real flow rate in the spinneret according to the company of $0.5 \mathrm{~mL} / \mathrm{h}$ ), $18 \mathrm{kV}$ of applied voltage and a $10 \mathrm{~cm}$ distance from the tip of the needle to the collector. These conditions ensured perfect polymer jets (continuous and free of droplets) for all spinning solutions. The fiber mats were produced at $50{ }^{\circ} \mathrm{C}$ after being collected onto the drum covered with non-sticky aluminum foil and rotating at $40 \mathrm{rpm}$.

\section{SEM}

The electrospun nanofibers were coated with a thin film of gold after being mounted onto specimen stubs, and the edge painted with colloidal silver adhesive prior to examine by Scanning Electron Microscopy (SEM; FEI, Hillsboro, OR). Photos were taken in the high-vacuum/secondary electron imaging mode using an accelerating voltage of $10 \mathrm{kV}$ and working distances between 10.2-11.8 $\mathrm{mm}$. At least one hundred fiber sizes were measured in the fibers shown in each SEM micrograph using the image analysis software XT Document (FEI Corp, Hillsboro, OR) to build a FDS histogram. 


\section{Statistical analysis}

The Shapiro-Wilk W test adapted to large sample sizes by [22] was used for testing normality $(P<0.05)$. Means of FDS following the normality and homogeneity of variance requirements were compared using the Tukey test $(P<0.05)$ while the Mann-Whitney test $(P<0.05)$ was used for comparing means of FDS following nonnormal distributions. One-way Analysis Of Variance (ANOVA) and Tukey test were performed to determine the significant differences between each mean value of surface tension, defined for $P<0.05$. All statistical analyses were performed using the software Statistica 8.0 (StatSoft Inc., Tulsa, OK).

\section{Results and discussion}

\section{Characterization of spinning solutions}

Rheological properties of the spinning solutions were measured through oscillatory tests to determine the dependence of viscoelastic properties with frequency and through steady state experiments to determine the solution viscosity as a function of shear rate. Ideally we should measure the solution behavior under extensional deformation since extrusion processes like electrospinning typically involve the polymer solution or melt stretching. However, elongational viscosity is much harder to measure and since it is proportionally related with shear viscosity, measurements under shear deformation are most commonly used to support electrospinning studies [7, 28].

Fig. 1 shows the viscoelastic properties (elastic (G') and viscous (G") moduli) at $50{ }^{\circ} \mathrm{C}$ of representative GG/CS28 (A) and GG/CS50 (B) blends, and of the GG alone, recorded from 0.01 to $10 \mathrm{~Hz}$. Pure starches are not plotted since it was hard to obtain meaningful data. Similar problems have been reported by other authors [2].

The spinning solutions showed G' and G' values that were frequency-dependent and varied up to several orders of magnitude within the range of studied frequencies. The GG alone ( $3 \mathrm{wt} \%$ ) showed the highest values of $G^{\prime}$ and $G^{\prime \prime}$ with the curves exhibiting a cross-over point around $0.1 \mathrm{~Hz}$ (i.e. $\mathrm{G}^{\prime}=\mathrm{G}^{\prime \prime}$ ), beyond which the viscoelastic behavior became predominantly elastic (G'> G') (Fig. 1). Similar behavior was observed by Torres et al. when studying GG solutions with concentrations above $1 \mathrm{wt} \%$ at $25{ }^{\circ} \mathrm{C}$ [32]. Despite the scattered data, it was clear a predominant elastic behavior ( $G^{\prime}>G^{\prime \prime}$ ) in the mechanical spectra of both starches (not shown). The magnitudes of the viscoelastic parameters were very low, between $\sim 0.0007-4 \mathrm{~Pa}$, especially at low frequencies.

Blends of GG/CS exhibited intermediate behaviors to those of the pure polysaccharides. For both CS, the elastic plateau $\left(G^{\prime}=G^{\prime \prime}\right)$ moved gradually to higher frequencies with the decrease of GG content in the blends (e.g. $\sim 0.3 \mathrm{~Hz}$ for $1: 1 \mathrm{GG} / \mathrm{CS} 28$ and $\sim 5$ $\mathrm{Hz}$ for 1:4 GG/CS28; Fig. 1A). Decreasing the GG concentration decreased the viscoelastic parameters and the curves approached those of the pure starches. The frequency-dependence of $G^{\prime}$ and G" was more significant for the pure CS and for the blends with higher CS contents. For instance, the $G^{\prime}$ values of GG/CS50 at 1:4 ratio varied from 0.01 to $10 \mathrm{~Pa}$ and the G" from 0.1 to $10 \mathrm{~Pa}$ while at $4: 1$ ratio, both moduli varied only by one order of magnitude (Fig. 1B).

Example flow curves at $50^{\circ} \mathrm{C}$ of the GG/CS blends and of the GG alone are represented in Fig. 2 for CS28 (A) and CS50 (B). Results for the pure CS were unreliable under the tested conditions and are not represented. The GG solution was highly viscous and showed a strong shear thinning behavior after a critical shear rate around $0.01 \mathrm{~s}^{-1}$ with the apparent viscosity decreasing over several order of magnitudes with increasing shear rates (Fig. $2 \mathrm{~A}$ ). Below $\sim 0.01 \mathrm{~s}^{-1}$, the solution exhibited a Newtonian plateau as previously observed for similar GG solutions [32] and other galactomannans [25]. Partial substitution of GG by CS28 or CS50 let to different flow behaviors with the viscosity and shearthinning effect gradually decreasing with the GG concentration.

\section{Correlation between rheology and fiber formation}

Spinning solutions prepared at $3 \mathrm{wt} \%$ total polysaccharide concentration and different GG/CS ratios were electrospun at 50 ${ }^{\circ} \mathrm{C}$ using predetermined electrospinning conditions. Preliminary tests showed that either CS produced blends that could be electrospun over a wide range of parameters without altering significantly the fiber morphology (not shown). Representative SEM images and FDS histograms of the obtained nanofibers are shown in Figs. 3 and 4 for the GG/CS28 blends and in Figs. 5 and 6 for the GG/CS50 blends.

Initially, the $3 \%$ GG solution flowed at the rate of $1 \mathrm{~mL} / \mathrm{h}$ but led to frequent needle clogging and discontinuous spinning with
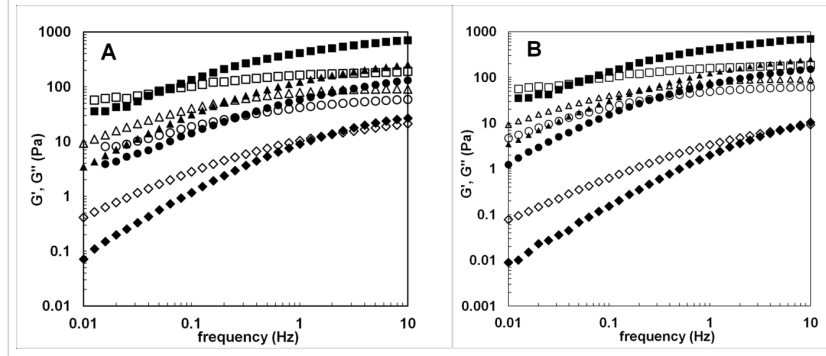

Figure 1: Frequency dependence at $50{ }^{\circ} \mathrm{C}$ of elastic (G'; filled symbols) and viscous (G"; open symbols) moduli of GG/CS28 (A) and GG/CS50 (B) blends prepared at $3 \mathrm{wt} \%$ concentration. Symbols $\rightarrow$ 1:0 (squares), 2:1 (triangles), 1:1 (circles),1:4 (diamonds) and GG/CS mass ratios. The measurements were recorded in the linear viscoelastic region of the samples.
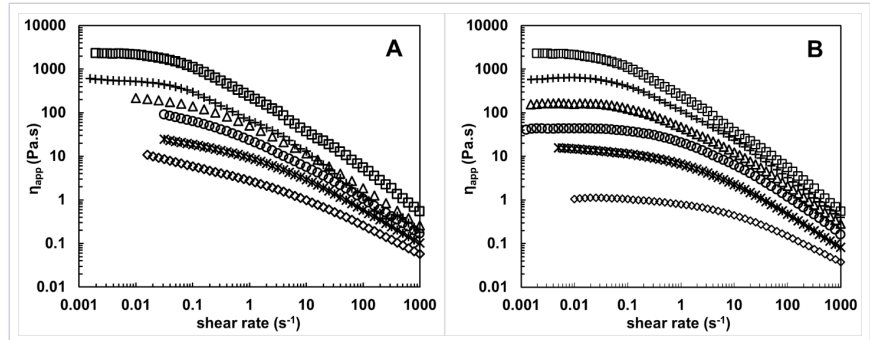

Figure 2: Flow curves at $50^{\circ} \mathrm{C}$ of GG/CS28 (A) and GG/CS50 (B) blends. Symbols $\rightarrow$ 1:0 (squares), 4:1 (plus signs), 2:1 (triangles), 1:1 (circles), $1: 2$ (asterisk) and 1:4 (diamonds) GG/CS mass ratios. 
the increase of spinning time. This limited spinnability agreed well with the high solution viscosity (Fig. 2) and predominant elastic behavior (Fig. 1) measured during the rheological tests. The pure galactomannan formed irregular nanofibers with smaller (2) and wider (1) FDS between 20 and $80 \mathrm{~nm}$ (Fig. $3 \mathrm{~A}$ ) which could be formed due to jet instabilities related with high solution viscosity $[4,31]$. In electrospinning, continuous and defect-free nanofibers with uniform FDS are formed from polymer solutions with adequate properties. While polymer concentration should ensure sufficient molecular entanglements to form a continuous polymer jet, the deformation of the solution droplet prior to jet formation must come from an adequate electrostatic force whose magnitude and stability will be determined by the solution viscosity, viscoelasticity, surface tension and electrical conductivity $[27,28,31]$. Due to the nature of our solutions the surface tension and electrical conductivities could not be measured but we believe that the rheological properties which are governed by molecular weight, molecular weight distribution, concentration of polysaccharide and solvent system will be determinant factors for fiber formation.

Similar to the results of another group [28], it was not possible to obtain fibers when electrospinning either CS (not shown). It is believed that starch fiber-forming ability is mostly linked to its amylose content since the highly branched nature of amylopectin cannot be as easily aligned and stretched to form continuous fibers [12]. While starch gelatinization is expected to occur below $100{ }^{\circ} \mathrm{C}$ (heating temperature of our starch dispersions) the transition from an helical to random coil conformation of starch molecules, which favors fiber formation, will only occur $\sim 160{ }^{\circ} \mathrm{C}$ [12]. For this reason, native starch fibers have only been obtained by electro-wet-spinning technology from DMSO solutions [10,11, $12,13]$ while electrospinning has only been possible using starch derivatives $[15,34]$ or starch blends with synthetic polymers [29, 30].

The GG/CS28 blend prepared at 4:1 ratio, containing $2.4 \mathrm{wt} \%$ GG and 0.6 wt $\%$ CS28, had viscosities (Fig. 2A) and viscoelastic properties (not shown) closer to those of the pure GG showing the same limitations in spinnability. Two populations of fibers were also formed (identified by 1 and 2 in Fig. 3B) with similar average FDS $(56 \pm 36 \mathrm{~nm})$ to that of the GG alone $(P>0.05)$ but in this case, the smaller fibers showed significant branching (2). Increasing the CS28 content in the blend from 0.6 to 1 $\mathrm{wt} \%$ significantly improved the jet stabilities and led to more uniform fiber morphology and to an increase in fiber size $(95$ $\pm 27 \mathrm{~nm}$; $P<0.05$; Fig. $3 \mathrm{C}$ ). A transition was also noted in the distributions of FDS from non-normal (Figs. $3 \mathrm{~A}$ and $3 \mathrm{~B}$; $P<0.05$ ) to normal $(P>0.05$; Fig. $3 \mathrm{C})$. GG solutions with concentrations between $1-2.4 \%$ were also electrospun under the same process parameters and compared with GG/CS blends. A 2\% GG solution showed good spinnability but the produced nanofibers showed some minor defects (Fig. 4B) proving that the addition of $1 \%$ CS28 was important to improve fiber morphology as shown in Fig. $4 \mathrm{~A}$ at lower magnification. At deformation rates closer to those felt in the polymer jet $\left(1000 \mathrm{~s}^{-1}\right.$;) [7], the viscosity of 2:1 GG/CS28 was $\sim 0.17$ Pa.s (Fig. 2A). When further increasing the
CS28 content (>1 wt $\%$; Figs. 3D-F), fiber morphology and FDS became gradually more irregular changing from beaded fibers at $1.5 \mathrm{wt} \%$ (Fig. 3D for 1:1 GG/CS28) to beaded fibers with other defects at $2 \mathrm{wt} \%$ CS28 or higher (Figs. 3E and F for 1:2 and 1:4 $\mathrm{GG} / \mathrm{CS} 28)$. The viscosities in the range 0.12-0.06 Pa.s (1000 s${ }^{-1}$; Fig. $2 \mathrm{~A}$ ) decreased with the increase in starch content and the FDS decreased down to a minimum average value of $33 \pm 13 \mathrm{~nm}$ for 1:4 GG/CS28 (Fig. 3F).

Blends prepared with CS50 showed similar spinnability to those prepared with CS28 and formed fibers with higher FDS $(P<0.05)$ except, the $\mathrm{GG} / \mathrm{CS} 50$ at $1: 4$ ratio $(32 \pm 10 \mathrm{~nm} ; P>0.05$; Fig. 5E). GG/CS50 at 4:1, with viscosities closer to those of the GG alone (Fig. 2B), could not form a continuous polymer jet (not shown). This blend formed nanofibers with a few beads and mean FDS of $89 \pm 17 \mathrm{~nm}$ (Fig. 5A).

Fibers with a few beads and FDS ranging from 89 to 127 $\mathrm{nm}$ were also formed from GG/CS50 2:1 (Fig. 5B). At an optimal ratio of $1: 1$ ( $1.5 \mathrm{wt} \%$ of GG and $1.5 \mathrm{wt} \%$ of CS50), the nanofibers became uniform and their FDS, with an average value of $81 \pm 14$ nm, normally distributed $(P>0.05$; Fig. $5 C)$. For this blend, the viscosity at $1000 \mathrm{~s}^{-1}$ was $\sim 0.16$ Pa.s (Fig. $2 \mathrm{~B}$ ), similar to that of

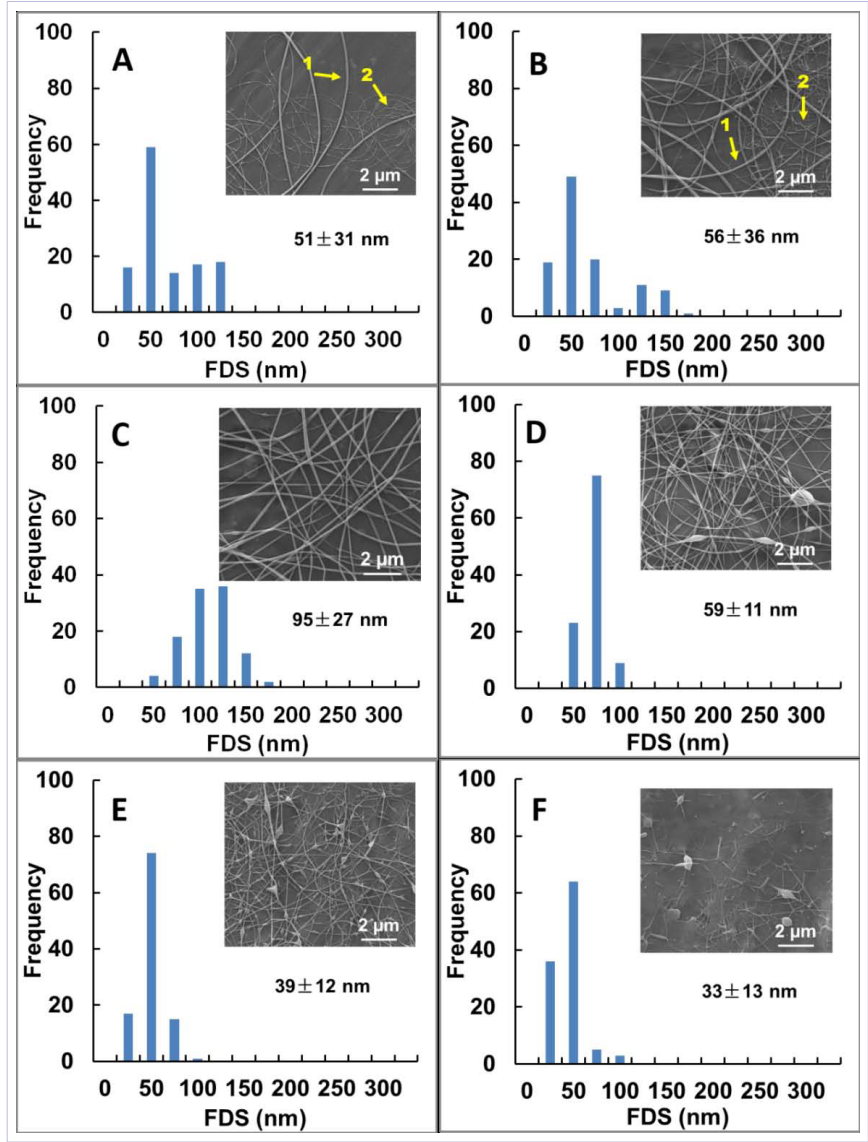

Figure 3: SEM images and fiber diameter size (FDS) analysis of electrospun nanofibers obtained from GG/CS28 blends with $3 \%$ wt total polysaccharide concentration and mass ratios of: (A) 1:0 (B) 4:1; (C) 2:1; (D) 1:1; (E) 1:2; (F) 1:4. Magnification is 25,000×. Numbers (1) and (2) indicate two populations of fiber sizes. 
the $2: 1 \mathrm{GG} / \mathrm{CS} 28$ that produced best nanofibers (Fig. 2A). A 1.5\% GG solution produced fibers with many defects (Fig. 6C) proving that the addition of CS50 facilitated the electrospinning process and led to more uniform fiber morphology (Fig. 6A). Contrarily to CS28 (not shown), the sonication of CS50 prior to mixing with GG significantly improved fiber morphology. The disintegration of starch granules leading to amylose leaching out into the aqueous phase takes place after the swelling of the granules caused by heat treatment. Contrarily to CS28, the CS50 was only

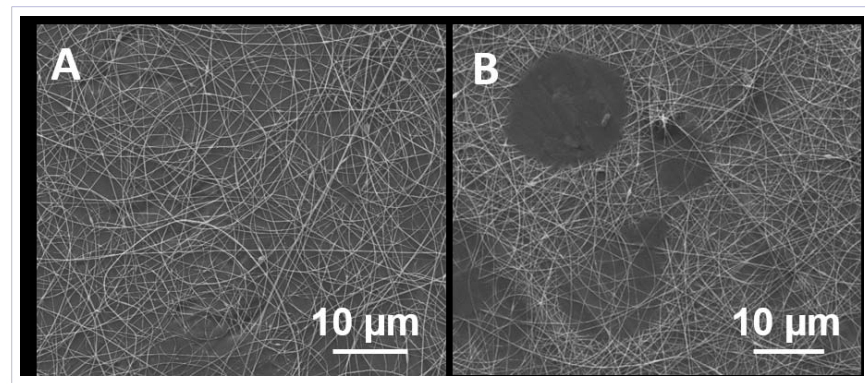

Figure 4: SEM images at lower magnification $(5,000 \times)$ of electrospun GG/CS28 nanofibers shown in Figure 3C corresponding, to 2:1 mass ratio (A). Nanofibers obtained from a $2 \%$ GG solution is shown in (B).

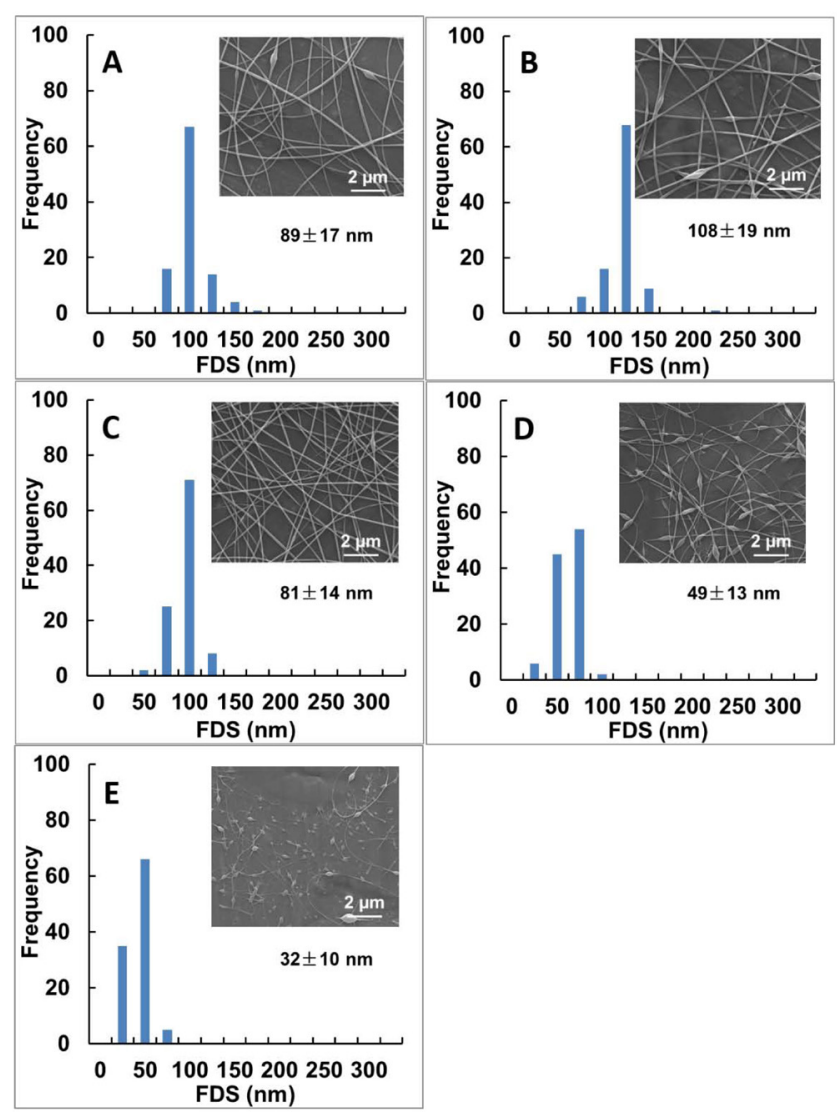

Figure 5: SEM images and fiber diameter size (FDS) analysis of electrospun fibers obtained from GG/CS50 blends with 3\% wt total polysaccharide concentration at $50^{\circ} \mathrm{C}$. Flow rate $=1 \mathrm{~mL} / \mathrm{h}$, Voltage $=18 \mathrm{kV}$. Mass ratios of GG/starch: (A) $4: 1$; (B) $2: 1$; (C) $1: 1$; (D) $1: 2$; (E) $1: 4$. Magnification is $25,000 \times$.
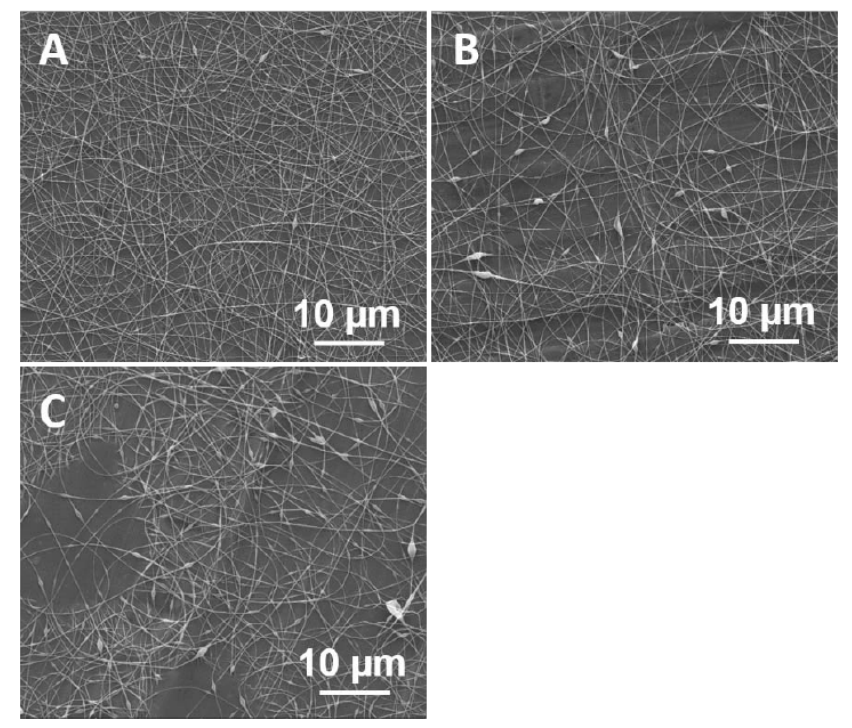

Figure 6: SEM images of electrospun nanofibers obtained at $50^{\circ} \mathrm{C}$ from: GG/CS50 blend with 3\% wt total polysaccharide concentration and mixed at 1:1 mass ratio with (A) and without (B) starch sonication. Nanofibers obtained from a $1.5 \%$ GG solution are shown in (C). Flow rate $=1 \mathrm{~mL} / \mathrm{h}$, Voltage $=18 \mathrm{kV}$. Magnification is $5,000 \mathrm{x}$.

partially gelatinized when heating the starch dispersions at 95 ${ }^{\circ} \mathrm{C}$ (gelatinization temperatures: $\sim 80{ }^{\circ} \mathrm{C}$ for CS28 and $\sim 103{ }^{\circ} \mathrm{C}$ for CS50 [21, 33]. We suspect that the sonication could stimulate the solubilization of CS50 in water leading to a better dispersion and separation of randomly aggregated amylopectin molecules, making the starch strands more homogeneous and aligned, which resulted in a significant improvement in fiber morphology $[9,23]$. An example of nanofibers produced with (A) and without starch sonication (B) is shown in Fig. 6 for the 1:1 GG/CS50. Increasing the starch content in the spinning solution from $1.5 \mathrm{wt} \%$ to 2 $w t \%$ led to the formation of nanofibers with many beads (Fig 5D) and non-normal distributions of FDS $(P<0.05)$. Mean FDS of GG/ CS50 1:2 decreased to $49 \pm 13 \mathrm{~nm}(P<0.05)$ which was not much different $(P>0.05)$ to that of the GG alone (Fig. 3A). A further increase in CS50 concentration to $2.4 \mathrm{wt} \%$ resulted in fibers with many defects as shown in Fig. 5E.

In the present study, defects in fiber morphology and limitations in spinnability of GG could be overcome by blending GG with CS with different amylose contents without the need for additional steps after GG purification. Differences in the optimal blending ratio of CS28 and CS50 with GG could be attributed to the different amylose contents and different preparation methods of the starches (CS50 was sonicated while CS28 was not).

\section{Conclusions}

Electrospun nanofibers were produced for the first time, from blends of GG and CS with different amylose contents. The CS were effective rheological modifiers that greatly improved the spinnability of GG solution. At an optimal viscosity and polysaccharide ratio, defect-free fibers could be formed from blends of either CS without the need for successive filtrations 
prior to electrospinning to eliminate GG aggregates in solution. The sonication of CS50 prior to mixing with GG significantly improved fiber morphology. GG has a long history of safe use as a component in food formulations, the present research showed the potential of GG as a carrier polymer for electrospinning fine fibers and fibrous mats from proteins and non-spinnable polysaccharides, such as pectin and chitosan, etc. for food-grade applications.

\section{Acknowledgements}

The authors thank Mr. Joseph Uknalis from the USDA, ARS, ERRC, Wyndmoor, PA, for the SEM analysis. W. Yang also acknowledges support of the program $12^{\text {th }}$ Five-Year Plans for National ST Development (No 2015BAD16B02).

\section{References}

1. Aceituno-Medina M, Mendoza S, Lagaron JM, López-Rubio A Development and characterization of food-grade electrospun fibers from amaranth protein and pullulan blends. Food Research International. 2013;54(1):667-674.

2. Ahmed J, Singh A, Ramaswamy HS, Pandey PK, Raghavan GSV Effect of high-pressure on calorimetric, rheological and dielectric properties of selected starch dispersions. Carbohydrate Polymers. 2014;103:12-21.doi: 10.1016/j.carbpol.2013.12.014

3. Be Miller JN. Pasting, paste, and gel properties of starch-hydrocolloid combinations. Carbohydrate Polymers. 2011;86(2):386-423.

4. Deitzel JM, Kleinmeyer J, Harris D \& Tan NCB. The effect of processing variables on the morphology of electrospun nanofibers and textiles. Polymer. 2001;42(1):261-272.

5. Ghorani B, Tucker N. Fundamentals of electrospinning as a nove delivery vehicle for compounds in food nanotechnology. Food Hydrocolloids. 2015;51:227-240.

6. Greiner A, Wendorff JH. Electrospinning: A fascinating method for the preparation of ultrathin fibres. Angewandte Chemie-International Edition. 2007;46(30):5670-5703.

7. Han T, Yarin AL, Reneker DH. Viscoelastic electrospun jets: initial stresses and elongational rheometry. Polymer. 2008;49(6):16511658.

8. Heyman B, De Vos, WH, Depypere F, Van der Meeren P, Dewettinck K. Guar and xanthan gum differentially affect shear induced breakdown of native waxy maize starch. Food Hydrocolloids. 2014;35:546-556.

9. Iida Y, Tuziuti T, Yasui K, Towata A, Kozuka T. Control of viscosity in starch and polysaccharide solutions with ultrasound after gelatinization. Innovative Food Science and Emerging Technologies. 2008;9:140-146.

10. Kong L, Ziegler G. Quantitative relationship between electrospinning parameters and starch fiber diameter. Carbohydrate Polymers. 2013;92(2):1416-1422.

11. Kong L, Ziegler G. Rheological aspects in fabricating pullulan fibers by electro-wet-spinning. Food Hydrocolloids. 2014;36:220-226

12. Kong L, Ziegler GR. Fabrication of pure starch fibers by electrospinning. Food Hydrocolloids. 2014;36:20-25.

13. Kong LY, Ziegler GR. Role of Molecular Entanglements in Starch Fiber Formation by Electrospinning. Biomacromolecules. 2012;13(8):22472253. doi: $10.1021 / \mathrm{bm} 300396 \mathrm{j}$

14. C,Arrechi A, KitK,McClementsDJ,Weiss J.Fabrication,functionalization, and application of electrospun biopolymer nanofibers. Critical Reviews in Food Science and Nutrition. 2008;48(8):775-797.

15. Lancuški A, Vasilyev G, Putaux JL, Zussman, E. Rheological Properties and Electrospinnability of High-Amylose Starch in Formic Acid. Biomacromolecules. 2015;16(8):2529-2536. doi: 10.1021/acs. biomac. 5 b00817.

16. Lubambo AF, de Freitas RA, Sierakowski MR, Lucyszyn N, Sassaki GL, Serafim BM, et al. Electrospinning of commercial guar-gum: Effects of purification and filtration. Carbohydrate Polymers. 2013;93(2):484491. doi: 10.1016/j.carbpol.2013.01.031

17. Meng F, Liang Y, Shuai T, Xiong J, Zhong G. Physicochemical properties of Amorphophallus paeoniifolius (Dennst.) Nicolson starch and its blend with konjac glucomannan. Starch/Stärke. 2015;67(9-10):820828.

18. Miles MJ, Morris VJ, Orford PD, Ring SG. The roles of amylose and amylopectin in the gelation and retrogradation of starch. Carbohydrate Research. 1985;135(2): 271-281.

19. Morell P, Fiszman SM, Varela P, Hernando I. Hydrocolloids for enhancing satiety: Relating oral digestion to rheology, structure and sensory perception. Food Hydrocolloids. 2014;41,343-353.

20. Murray BS, Phisarnchananan, N. The effect of nanoparticles on the phase separation of waxy corn starch + locust bean gum or guar gum. Food Hydrocolloids. 2014;42:92-99.

21.S, Vasanthan T, Hoover R, Bressler D. The susceptibility of large and small granules of waxy, normal and high-amylose genotypes of barley and corn starches toward amylolysis at sub-gelatinization temperatures. Food Research International. 2013;51(2):771-782.

22. Royston JP. An extension of Shapiro and Wilk's W test for normality to large samples. Applied Statistics. 1982;31(2):115-124.

23. Seguchi M, Higasa T, Mori T. Study of wheat starch structures by sonication treatment. Cereal Chemistry. 1994;71(6):636-639.

24. Sittikijyothin W, Torres D, Gonçalves MP. Modelling the rheological behaviour of galactomannan aqueous solutions. Carbohydrate Polymers. 2005;59(3):339-350.

25. Sousa AMM, Gonçalves MP. Strategies to improve the mechanical strength and water resistance of agar films for food packaging applications. Carbohydrate Polymers. 2015;132:196-204. doi: 10.1016/j.carbpol.2015.06.022.

26. Sousa AMM, Souza HKS, Uknalis J, Liu SC, Gonçalves MP, Liu L. Electrospinning of agar/PVA aqueous solutions and its relation with rheological properties. Carbohydrate Polymers. 2015;115:348-355.

27. Sousa AMM, Souza HKS, Uknalis J, Liu SC, Gonçalves MP, Liu L. Improving agar electrospinnability with choline-based deep eutectic solvents. International Journal of Biological Macromolecules. 2015;80:139-148. doi: 10.1016/j.ijbiomac.2015.06.034.

28.Stijnman AC, Bodnar I, Tromp RH. Electrospinning of food-grade polysaccharides. Food Hydrocolloids. 2011;25(5):1393-1398.

29. Sukyte J, Adomaviciute, E, Milasius R. Investigation of the possibility of forming nanofibres with potato starch. Fibres \& Textiles in Eastern Europe. 2010;18(5):24-27.

30.Sunthornvarabhas J, Chatakanonda P, Piyachomkwan K, Sriroth K. Electrospun polylactic acid and cassava starch fiber by conjugated solvent technique. Materials Letters. 2011;65(6):985-987.

31. Tomasula PM, Sousa AMM, Liou SC, Li R, Bonnaillie LM, Liu LS. Electrospinning of casein/pullulan blends for food grade applications. 
Journal of Dairy Science. 2016;99(3):1837-1845. doi: 10.3168/ jds.2015-10374

32. Torres MD, Hallmark B, Wilson DI. Effect of concentration on shear and extensional rheology of guar gum solutions. Food Hydrocolloids. 2014;40:85-95.

33. Vamadevan V, Hoover R, Bertoft E, Seetharaman K. Hydrothermal treatment and iodine binding provide insights into the organization of glucan chains within the semi-crystalline lamellae of corn starch granules. Biopolymers. 2014;101(8):871-885.

34.Xu W, Yang W, Yang Y. Electrospun starch acetate nanofibers: Development, properties, and potential application in drug delivery. Biotechnology Progress. 2009;25(6):1788-1795. doi: 10.1002/ btpr.242. 